\title{
Enfermedades Autoinmunes, tratamiento con Trichuris suis y otros helmintos
}

\author{
Autoimmune diseases, treatment with Trichuris suis and some other helminths \\ Iñigo Pallardo Fernández \\ Graduado en Farmacia en la Universidad Complutense de Madrid en 2014
}

\author{
Artículo de Revisión \\ Review Article \\ Correspondencia \\ Correspondence \\ Iñigo Pallardo Fernández, \\ móvil +34608387130 \\ inigo-pallardo@cofm.es, \\ C/Nelázquez 136, 9² A, 28006 Madrid. \\ Financiación \\ Fundings \\ Trabajo realizado sin financiación.

\section{Conflicto de interés} \\ Competing interest \\ No hay conflicto de intereses \\ Agradecimientos \\ Acknowledgements \\ Al profesor Francisco Bolás, catedrático \\ del Departamento de Parasitología de la \\ Facultad de Farmacia de la Universidad \\ Complutense de Madrid, bajo cuya di- \\ rección he realizado el presente trabajo \\ y aprendido mucho sobre la materia.
}

Received: 15.09.2014 Accepted: 02.02.2015

\section{RESUMEN}

Objetivos. La "Hipótesis de la Higiene" postula sobre los efectos inmunomoduladores inducidos por agentes infecciosos en los seres humanos. El objetivo principal de este trabajo es indagar sobre las evidencias de esa hipótesis y sobre sus aplicaciones en el campo del tratamiento de las enfermedades autoinmunes, haciendo especial hincapié tanto en los mecanismos de acción en los que se basan estas aplicaciones como en los resultados reales obtenidos. Además analizará la posible evolución de estas terapias, especialmente a la sombra de las controversias éticas que surgen de la aplicación de estos tratamientos en relación a si es lícito causar una infección para curar otra patología, como es el caso que nos ocupa.

Resultados. Actualmente existen resultados prometedores de ensayos clínicos sobre terapias helmínticas aplicadas al tratamiento de enfermedades autoinmunes como son la enfermedad de Crohn y la colitis ulcerosa, resumidos en el apartado de resultados de este artículo.

Igualmente prometedora es la gran variedad de ensayos clínicos que actualmente están en curso sobre la aplicación de la terapia helmíntica al tratamiento de diversas patologías en las que está involucrado el sistema inmunológico, como son: asma, rinitis alérgica, artritis reumatoide, esclerosis múltiple, diabetes Mellitus tipo I, encefalomielitis autoinmune, obesidad, autismo, etc que han llevado a identificar cuáles son los parásitos indicados en el tratamiento de este amplio espectro de enfermedades.

Sin embargo es necesario indicar que no todos los helmintos son inmunorreguladores $y$, por lo tanto útiles en el tratamiento de estas enfermedades y que, los que lo son, no son útiles en el tratamiento de todas las enfermedades de origen inmunológico sino que presentan una marcada especificidad. Es más, la utilidad de éstos presenta una variabilidad importante, no sólo dependiente de la enfermedad de origen inmunológico a tratar, sino también de las condiciones del paciente. Es por ello que actualmente no existen terapias helmínticas aprobadas por las principales agencias del medicamento; aún quedan muchos aspectos por desvelar, lo que hace que la hipótesis de la higiene no haya pasado de ser una hipótesis. Sin embargo lo prometedor de estas terapias ha traído consigo la autorización de algunas de ellas como "productos en fase de investigación clínica".

Conclusiones. Es necesario seguir investigando sobre las terapias helmínticas. Además es especialmente prometedor el empleo de productos solubles de parásitos en el tratamiento de estas enfermedades en lugar de la infección helmíntica e incluso, el diseño de análogos sintéticos que sean más efectivos y presenten menos riesgos. La utilización de este último tipo de productos traerá consigo un aumento en el número de ensayos clínicos, justificados por las facilidades de patente de éstos frente a la utilización directa de estados de los parásitos. Sin embargo todavía es necesaria más investigación para conocer las biomoléculas presentes en los parásitos que son responsables de su efecto terapéutico.

Palabras Clave: enfermedades autoinmunes, hipótesis de la higiene, terapia con helmintos, Trichuris suis. 


\section{ABSTRACT}

Objectives. Hygiene hypothesis postulates about immunomodulatory effects induced by some infectious agents in human beings. The main objective of this review is to find out what the evidence of this hypothesis is and its applications in the field of autoimmune diseases, paying especial attention not only in the action mechanisms in which they are based upon but also in the real outcome obtained. Moreover, the likely evolution of these therapies will be analyzed, especially in what is related to ethical disputes arising from these treatment applications regarding whether the causing infection is fair in order to heal another pathology, as the case may be.

Outcome. Promising outcomes currently exist in regards to helminth therapy applied to treat autoimmune diseases such as Crohn's disease and ulcerative colitis, summarised in this review.

Equally promising is the high amount of outstanding clinical trials for the application of helminthic therapy to a wide range of different pathologies in which the immune system is involved, such as: asthma, allergical rhinitis, rheumatoid arthritis, type I diabetes mellitus, autoimmune encephalomyelitis, obesity, autism, etc, which have identified useful parasites for the treatment of such a wide spectrum of diseases.

However it is needed to highlight that helminths are not always immunoregulators and therefore useful in all autoimmune disease treatments. Those which are immunoregulators, are not useful in any autoimmune disease treatment because they are highly specific. Moreover, its usefulness shows high variability, which means it is not only dependent on disease but also dependent upon patient conditions. It is because of this that helminthic therapies are not currently approved by major medical agencies; still many outstanding aspects need to be unveiled, because hygiene hypothesis has not overcome the hypothesis status. However these promising therapies have gained the status of "investigational products".

Conclusions. Additional investigation is needed. Especially promising are parasitic soluble products employed in autoimmune disease treatments in place of helminthic infection and even, less risky and more effective new analogue synthetic drug designs. This latter kind of product utilisation will increase the number of clinical trials due to patent ease of this product versus direct utilisation of parasitic phases. However more investigation is still needed to know which parasitic biomolecules are responsible for the therapeutic effect.

Keywords: autoimmune diseases, hygiene hypothesis, helminthic therapy, Trichuris suis.

\section{INTRODUCCIÓN}

El objetivo del presente artículo esanalizar las actuales técnicas de tratamiento de enfermedades autoinmunes con helmintos en basea la revisión bibliográfica sobre ensayos clínicos e investigación científica en los campos de la inmunología, la parasitología y la farmacología, ver epígrafe de Materiales y Métodos. La aportación del artículo es presentar estas técnicas en un marco amplio, relacionándolas tanto entre sí como con aspectos de la teoría evolutiva de parásitos, de la hipótesis de la higiene, de las enfermedades autoinmunes y de las tecnologías de preparación de estos biofármacos; de este modo se estará en condiciones de entenderpor qué la utilización de helmintos supone una oportunidad segura y eficaz en el tratamiento de este tipo de enfermedades, y discutir cuáles podrían ser los próximos pasos en el desarrollo de las mismas. Para entender cuáles son los motores que pueden dirigir la evolución futura de las terapias helmínticas también se tomarán en consideración aspectos económicos y éticos.

En general, las enfermedades autoinmunes presentan una enorme casuística, teniendo en común el hecho de producir daño a tejidos y órganos por medio de la respuesta Th1 y Th17 del sistema inmunológico, inducida por el reconocimiento de autoantígenos. En general estas enfermedades, además de los síntomas locales derivados del daño a los tejidos en su órgano diana, van acompañadas en muchas ocasiones de otras enfermedades autoinmunes que afectan a otros órganos, como son distintos tipos de artritis, lesiones cutáneas con eritema, anemias de origen autoinmune, etc.

Este tipo de enfermedades en su conjunto afectan a todos los grupos etarios, desde la niñez hasta la senectud, siendo muchas de ellas muy específicas de determinadas edades. Por otro lado, y también en términos generales, presentan una fuerte asimetría por sexo, teniendo una mucha mayor incidencia en mujeres.

En su padecimiento no sólo son relevantes los aspectos genéticos, que son los determinantes de la predisposición a padecerlas, sino que también lo son los ambientales en cuanto a la presentación de las mismas, entre los que destaca la adquisición de infecciones, que impacta sobre la presentación de enfermedades autoinmunes endos sentidostotalmente contrapuestos:

- Por un lado, facilitando su presentación en aquéllos casos en los que el individuo muestrauna predisposición genética, por ejemplo: Guillain-Barré en relación con Campylobacter yeyuni o con el virus Epstein Barr; Diabetes mellitus tipo I con Cocksakie B4; Fiebre reumática con Streptococcus; enfermedades inflamatorias intestinales con Chlamydia y Mycobacteria.

- Por otro lado, modulando la respuesta autoinmune en individuos afectados por enfermedades autoinmunes, por ejemplo en el caso de la adquisición de determinadas infecciones gastrointestinales causadas por helmintos.Esto es lo que postula la hipótesis de la higiene ${ }^{(1)}$.

Ambos sentidos muestran la enorme relación de las enfermedades autoinmunes con las infecciones, estando basado el segundo de ellos en la Hipótesis de la Higiene, que postula cómo determinados parásitos, a través del proceso de 
co-evolución con sus hospedadores, clave en el éxito evolutivo de todo parásito, son capaces de ejecutar determinadas funciones de efecto inmunorregulador en sus hospedadores, siendo esta la clave de las terapias inmunorreguladoras basadas en helmintos que se introducen en este artículo.

¿En qué evidencias se basa la hipótesis de la higiene?:

- Correlación entre la distribución geográfica de las zonas con elevada prevalencia de enfermedades autoinmunes y sus buenas condiciones higiénico-sanitarias, que determinan una ínfima incidencia de enfermedades parasitarias, figura 1

- Correlación entre la distribución temporal de las enfermedades autoinmunes, en aumento, y la mejora en las condiciones higiénico - sanitarias, determinante de una reducción paralela de los casos de infecciones parasitarias.

- Experimentos realizados con modelos animales mantenidos en condiciones de esterilidad, con resultado de un aumento de incidencia de enfermedades autoinmunes y una reducción de la esperanza de vida.

- Correlación entre la distribución geográfica de las zonas con baja prevalencia de enfermedades autoinmunes y sus condiciones higiénico-sanitarias deficitarias, que determinan una elevada incidencia de enfermedades parasitarias, Figura 1.

Figura 1. En azul geografías de elevada incidencia de enfermedades autoinmunes. En rojo, geografías con elevada incidencia de infecciones helmínticas.

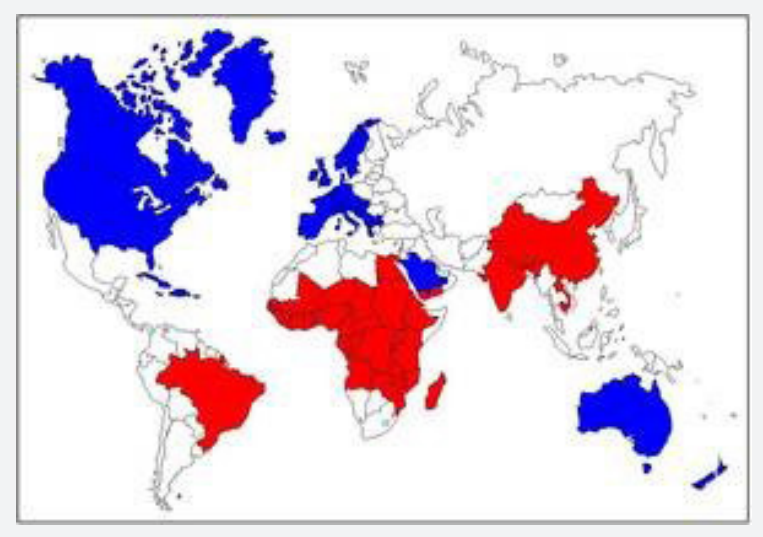

En relación con la Hipótesis de la Higiene surgen una serie de preguntas como: ¿Son todos los parásitos inmunorreguladores? ¿Es posible utilizarlos todos en la terapia de enfermedades autoinmunes, como las enfermedades inflamatorias intestinales? Como se verá más adelante, no todos los parásitos son inmunorreguladores, sino sólo aquéllos en los que el proceso de co-evolución que caracteriza la relación parásito hospedador, ha desembocado en una solución evolutiva de este tipo.
En relación con la segunda pregunta, no todos los parásitos inmunorreguladores pueden utilizarse en el tratamiento de enfermedades autoinmunes en humanos, ya que como se verá al analizar el caso concreto del empleo de Trichuris suis, son necesarios algunos requisitos adicionales a la inmunorregulación para que esto pueda hacerse de un modo seguro.

\section{MATERIAL Y MÉTODOS}

Se ha revisado diversa documentación constituida principalmente por las publicaciones internacionales más recientes y relevantes en los siguientes campos: mecanismos de acción de la regulación inmunitaria debidos al contacto con helmintos, posibles terapias utilizando helmintos, empleo de Trichuris suis como terapia en enfermedades autoinmunes, ensayos clínicos realizados con Trichuris suis, enfermedades autoinmunes, esclerosis múltiple, colitis ulcerativa, enfermedad de Crohn, enfermedad inflamatoria intestinal, otras enfermedades de origen inmunológico, parasitología evolutiva en nematodos, hipótesis de la higiene, etc. La tipología de la documentación revisada está constituida principalmente por "artículos" y "revistas científicas", tanto en español como en inglés, ya que el tipo de material de interés corresponde a mecanismos terapéuticos actualmente en investigación, sobre los que apenas hay datos en otro tipo de recursos como pueden ser libros de texto o similar. Toda la documentación revisada para este artículo es posterior a 2001, con especial atención a aquélla posterior a 2010. Las bases de datos consultadas para localizar artículos han sido, principalmente y entre otras: el catálogo CISNE, UCM - AECID, SciELO, PubMed, el catálogo de revistas extranjeras Bucea, y Google Scholar.

El autor además ha realizado algunas ponencias sobre el tema con el objetivo de recoger aportaciones de los participantes. Las exposiciones y comunicaciones orales se han llevado a cabo en la Universidad Complutense de Madrid.

A continuación se presentan los resultados de la revisión realizada orientados a los objetivos planteados, con el fin de presentar, difundir y explicar estas terapias desde el marco más amplio de la inmunología, la parasitología y la farmacología, así como concluir aspectos relevantes sobre ellas.

\section{RESULTADOS}

\section{Parásitos de utilidad}

La relación de las infecciones parasitarias con la respuesta del sistema inmunológico puede ser de diferente signo: inmunomoduladora, proinflamatoria, alérgica o neutra, dependiendo del parásito en cuestión además de otros condicionantes como son la edad del paciente, su fisiopatología 
y las condiciones de adquisición de la infección. El primero de los tipos de respuesta indicados es el que soporta la hipótesis de la higiene introducida más arriba. Evidentemente una de las condiciones que deben cumplirse para la terapia helmíntica es que el parásito que se emplee posea efectos inmunomoduladores. A continuación, tabla 1, se indican algunos parásitos de utilidad en terapia helmíntica de enfermedades autoinmunes así como en otras de origen inmunológico, algunos de ellos ya en ensayos clínicos y otros aún en fases experimentales ${ }^{(3,4)}$.

Tabla 1. Parásitos de utilidad como terapia helmíntica. Elaboración propia a partir de ${ }^{(3,4,5)}$

\begin{tabular}{|c|c|c|c|}
\hline Enfermedad & Parásitos & 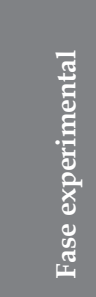 & 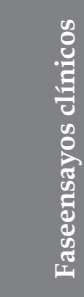 \\
\hline \multirow{2}{*}{$\begin{array}{l}\text { Enfermedad inflamatoria intestinal } \\
\text { (Enfermedad de Crohn y colitis ulcerativa) }\end{array}$} & T. suis, Trichuristrichiura, Necatoramericanus ${ }^{(5)}$ & & $x$ \\
\hline & $\begin{array}{l}\text { Hymenolepis diminuta, Schistosoma mansoni, Heligmoso- } \\
\text { moidespolygyrusbakeri, Trichinella spiralis }\end{array}$ & $x$ & \\
\hline \multirow{2}{*}{ Esclerosis múltiple } & T. suis & & $x$ \\
\hline & Strongyloides stercoralis,Ascaris lumbricoides & $x$ & \\
\hline \multirow[b]{2}{*}{ Alergia / Asma } & Necatoramericanus, T. suis & & $x$ \\
\hline & $\begin{array}{l}\text { Schistosoma mansoni, Trichinella spiralis } \\
\text { Acanthocheilonema viteae }\end{array}$ & $x$ & \\
\hline Celiaquía & Necatoramericanus & & $x$ \\
\hline Encefalomielitis autoinmune & Schistosoma mansoni, Trichinella spiralis, Fasciola hepatica & $x$ & \\
\hline Diabetes Melitus tipo I & $\begin{array}{l}\text { Schistosoma mansoni, Trichinella spiralis, Heligmosomoidespo- } \\
\text { lygyrusbakeri }\end{array}$ & $x$ & \\
\hline Artritis reumatoide & $\begin{array}{l}\text { Heligmosomoidespolygyrusbakeri, Nippostrongylusbrasiliensis, } \\
\text { Schistosoma mansoni, Acanthocheilonema viteae, Schistosoma } \\
\text { japonicum }\end{array}$ & $x$ & \\
\hline Obesidad & Nippostrongylusbrasiliensis & $x$ & \\
\hline
\end{tabular}

Además del tratamiento de enfermedades autoinmunes en sí, la terapia helmíntica se abre hacia el tratamiento de otras dolencias en las que esté involucrado el sistema inmunológico; a destacar por su elevada prevalencia están las alergias $\mathrm{y}$, por la no existencia de terapias con un binomio riesgo-beneficiosatisfactorio, el autismo.

En cuanto al autismo, puede resultar llamativo incluirlo aquí, sin embargo existen hallazgos sobre la alteración tanto absoluta como relativa, en los niveles de citoquinas relacionadas con las respuestas Th1 y Th2 del sistema inmunológico en los casos de autismo ${ }^{(6,7)}$. Además, y también en relación con el sistema inmunológico, existe evidencia sobre disfunciones en la barrera intestinal en el autismo que incrementan su permeabilidad ${ }^{(6,7)}$; esta disfunción posibilita el paso de antígenos derivados de la dieta que in- ducen reacciones del sistema inmunológico. La conexión entre estos hechos y la presentación de la patología autista está actualmente en estudio, pero apunta a la reacción del sistema inmunológico sobre antígenos del sistema nervioso. Es interesante recalcar cómo los patrones geográficos y temporales de la hipótesis de la higiene indicados anteriormente son igualmente válidos para la patología autista. Es por todo ello que el tratamiento del autismo con T. suis se está investigando actualmente.

\section{Condiciones que deben satisfacer los parásitos para ser utilizables en el tratamiento}

¿Puede concluirse del epígrafe anterior que cualquier parásito con efectos inmunomoduladores puede ser utilizado en este tipo de terapias? No, existiendo otros condicionantes igualmente importantes como son: 
- La posibilidad de producción del parásito o de sus componentes en laboratorio de un modo económico y que garantice la disponibilidad adecuada a la demanda esperada.

- La "inocuidad" del parásito. Significando aquí inocuidad la falta de inducción de condiciones patológicas y de transmisibilidad en el paciente pero no la inactividad, ya que el parásito debe realizar algún tipo de acción en el sistema inmune del paciente, que justificará su valor terapéutico. Más adelante se indicará en qué consisten estas acciones.

- No debe ser vector potencial de otras enfermedades. Evidentemente los productos a utilizar se someterán a procesos de purificación, aislamiento y axenización, ver figura 5 más adelante, que harán inviable la actuación de los mismos como vectores de otras enfermedades; sin embargo, por razones de duplicación de seguridad, es conveniente que sus posibilidades como vector de enfermedades humanas sean, en sí mismas, limitadas.

\section{Mecanismos de acción}

El efecto inmunomodulador en que se basa la utilización de helmintos en estas terapias se fundamenta en tres aspectos, que se esquematizan a través de la figura 2 que se muestra a continuación.

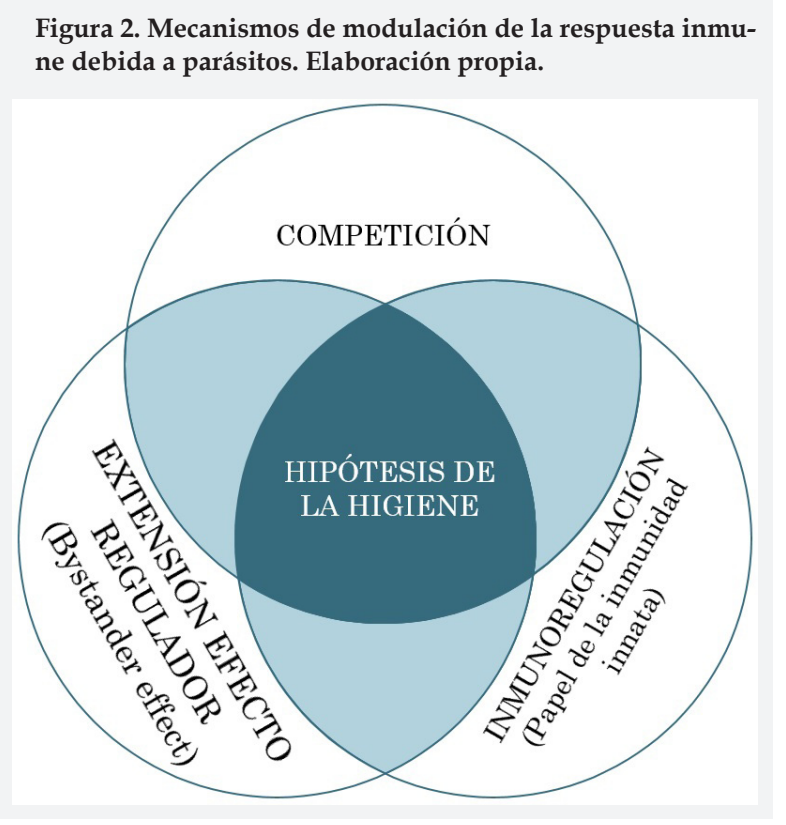

1. La ocupación de los recursos del sistema inmunológico en reaccionar frente al parásito que causa la infección hace que en los casos de patologías autoinmunes, la virulencia de las mismas disminuya ya que el sistema inmunológico dispondrá de menos recursos para reaccionar contra los autoantígenos, que son biomoléculas propias del paciente de enfermedad autoinmune contra las que actúa anormalmente su sistema inmunológico.

2. Inmunorregulación en el marco de la relación parásito/ hospedador. El éxito evolutivo de un parásito depende del proceso de co-evolución con su hospedador. En algunos casos, los que nos interesan en terapéutica, esta co-evolución se manifiesta a través de un fenómeno modulador del sistema inmunitario del hospedador que beneficia tanto al parásito, que tiene que hacer frente a una menor respuesta inmune, como al hospedador, que sufre menores efectos inflamatorios y patológicos, mejorando su calidad de vida y, por lo tanto siendo susceptible de alojar al parásito en mejores condiciones y durante más tiempo. Esta inmunorregulación está basada en diversos mecanismos que, dependiendo del parásito y del hospedador, tienen en común la relevancia del papel de la inmunidad innata en el proceso inmunorregulatorio. Para ilustrar esto se detalla a continuación el mecanismo de acción en el caso de T. suis. En este, los glicanos presentes en los productos solubles de T. suis son capaces de ligar con algunos tipos de receptores presentes en las células dendríticas (CD), como son:

- Receptor de lectina C. Este receptor, igual que el siguiente, se encarga del reconocimiento de determinados tipos patrones moleculares asociados a patógenos (PAMPs). La unión a sus ligandos produce por un lado, el incremento de la capacidad fagocitaria y tóxica microbiana de las propias CDs, y, por otro lado la producción de citoquinas que, a través de la unión a sus propios receptores son coadyuvantes en el desencadenamiento de respuestas de la inmunidad adaptativa.

- Receptor de manosa. Mismo caso que el anterior.

- Receptor Intercellular Adhesion Molecule 3 (ICAM-3 o también CD 50), que es una molécula de adhesión con dominios tipo inmunoglobulina que, además de sus funciones de adhesión, desempeña funciones de señalización a través de transducción de señales celulares al unirse a sus ligandos, como es LFA-1 presente en la membrana de los linfocitos. Esta unión forma parte de la "sinapsis inmunológica", coestimulando la unión del receptor del linfocito $\mathrm{T}$ (TCR)con elconjuntoantígeno/ complejo mayor de histocompatibilidad $(\mathrm{CMH})$ en la señalización responsable de la respuesta inmune adaptativa.

La unión de glicanos originarios de T. suis a los receptores indicados en lugar de los ligandos propios de estos receptores, induce cambios en el fenotipo de las CD que traen consigo:

- una inducción en la producción de citoquinas reguladoras y supresoras como TGF- $\beta$ e IL-10 y 
- una inhibición en la producción de citoquinas proinflamatorias como IL-6, IL-12, TNF- $\alpha$ e IFN- $\gamma$.

Este cambio en la composición del espectro de citoquinas expresado por las CD trae consigo, vía transducción de señales, cambios en el fenotipo y expresión de diferentes elementos celulares tanto de la inmunidad innata como de la adaptativa, como son ${ }^{(8)}$ :

- Viraje del perfil de respuesta de la inmunidad adaptativa desde Th1 y Th17, relacionados con enfermedades autoinmunes, hacia Th2, relacionado con otro tipo de patologías como las alergias ${ }^{(9)}$.

- Inducción de un mayor recuento de elementos celulares reguladores como son los linfocitos Treg y linfocitos B reguladores, que expresan IL-10.

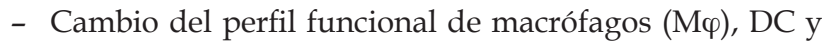
células T NK, cuya disfunción o defecto de recuento ha sido relacionado con numerosas enfermedades autoinmunes, alérgicas e incluso con procesos carcinogénicos.

El cambio fenotípico de estas células deriva en un viraje desde funciones fagocíticas y proinflamatorias hacia funciones reparadoras de tejidos que, en el caso de los M $\varphi$ tiene lugar a través de la vía de señalización celular IL-4Ra/ STAT6.

- Proliferación de las células epiteliales de la mucosa intestinal y diferenciación de las caliciformes debido a las acciones tróficas de IL-4 procedente de la respuesta Th2, lo que desemboca en una mayor producción de mucus. Estos hechos mejoran integridad y efectividad de la barrera intestinal frente a patógenos de todo tipo, reduciendo las respuestas inmunes derivadas de su permeabilidad.

3. Efecto espectador o extensión del efecto inmunorregulador explicado en el punto anterior a otros ámbitos más allá de la relación sistema inmunológico - parásito( ${ }^{(8,11)}$, efecto que aún no está bien documentado científicamente. Como se ha explicado en el punto anterior, existe un efecto regulador debido a la activación de la respuesta Th2 en detrimento de la Th1 debido a la infección del parásito; esto resulta en una atenuación de la morbilidad causada por la respuesta inmune sin que la inmunidad frente a infecciones sea atenuada, ya que estaría actuando la Th2, que produce menos daño e inflamación que la Th1. Pues bien, esteefectose refiere específicamente a la reacción de la inmunidad adaptativa frente a los antígenos parasitarios, que han sido los protagonistas en primera instancia de la inmunorregulación debida a parásitos vista en el punto anterior. Sin embargo esta regulación acabará afectando también a las relacionesespecíficas de la inmunidad adaptativa con otros antígenos distintos de los del parásito, denominados metafóricamente "espectadores" de todo el proceso, entre los que están los autoantígenos: biomoléculas responsables de la activación de la respuesta autoinmune, que es patológica. Es por ello que este efecto espectador produce finalmente una atenuación de los síntomas de las enfermedades autoinmunes.

Figura 3. Mecanismos inmunorreguladores comunes en parásitos inmunomoduladores, basado en el mecanismo de T.suis. Elaboración propia a partir de ${ }^{(3,10)}$.

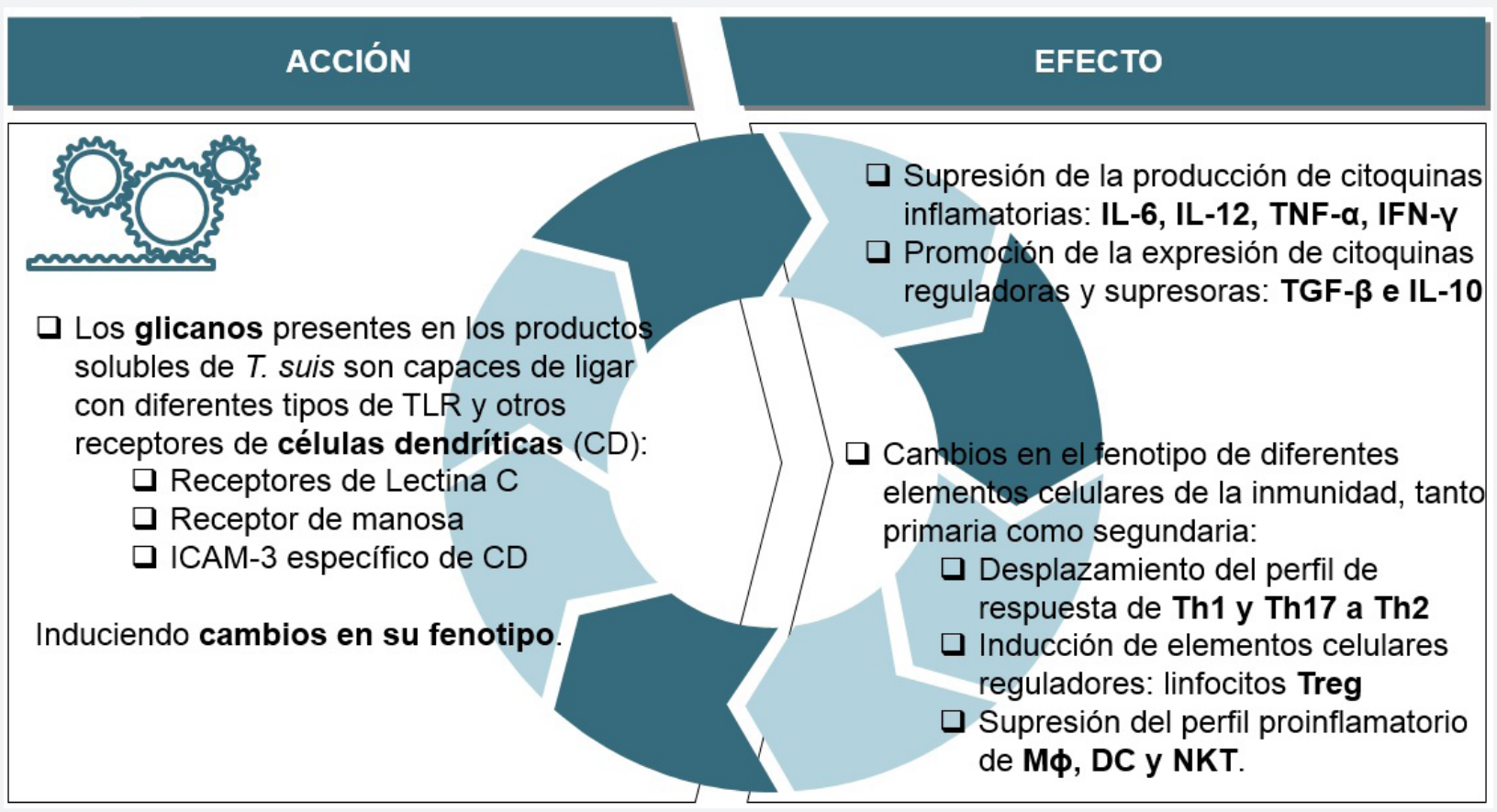


En resumen, el equipamiento biomolecular del parásito produce en el hospedador una atenuación de la virulencia de la respuesta inmunológica frente a él, que acabará regulando también la respuesta inmunológica frente a otros antígenos, como son los responsables de la autoinmunidad; esto produce una mejoría en los síntomas de las enfermedades autoinmunes.

A pesar de que la respuesta del sistema inmunológico a la presencia de helmintos es en general la respuesta alérgica, existe oportunidad en el tratamiento de alergias por medio de algunas especies de helmintos, a través de un mecanismo basado en los mismos preceptos que las enfermedades autoinmunes pero, en este caso, reduciendo la severidad de la respuesta Th2, cuya disfunción es responsable de las patologías alérgicas. Así las glicoproteínas ricas en fosforilcolina presentes en los productos solubles de Acanthocheilonema viteae, pueden interferir la respuesta alérgica mediante bloqueo de la activación de los mastocitos vía unión al receptor $F_{c}$ de alta afinidad, reduciendo de este modo tanto la liberación de citoquinas proinflamatorias como la degranulación de mediadores tóxicos de los mastocitos. Incluso actualmente se ha ido un paso más allá desarrollando análogos sintéticos de esta glicoproteína ES-62, concretamente de los restos de fosforil-colina que presenta ${ }^{(9)}$, ver figura 4. En las conclusiones se indica la relevancia de esta línea de actuación en relación con la viabilidad económica de la realización de investigación clínica en este campo.

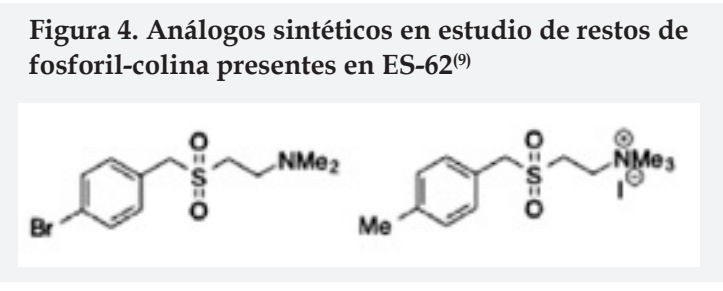

Por otro lado, de las indicaciones dadas sobre el autismo en el epígrafe sobre "parásitos de utilidad" en relación con la implicación del sistema inmunológico en la enfermedad, se sigue de este epígrafe la teórica utilidad de la terapia biológica del autismo con T. suis, actualmente en investigación.

\section{Utilización de Trichuris suis}

\section{Biología del parásito}

Trichuris suis es un parásito muy próximo a Trichuris trichiura, que es vulgarmente conocido como "gusano látigo", "whipworm" o "tricocéfalo", pero que parasita a determinados suidos en lugar de al hombre. Morfológicamente es indiferenciable de T. trichiura, habiendo que recurrir a técnicas moleculares, como PCR, para su diferenciación ${ }^{(12)}$.
¿Cómo funciona Trichuris suis en el hombre? Se le supone un ciclo biológico incompleto o interrumpido: en el ser humano los huevos embrionados llegan a eclosionar en el intestino, pudiendo las larvas L1 producir una colonización muy corta y autolimitada, asintomática, pero suficiente para inducir la respuesta moduladora buscada en el sistema inmunológico. Sin embargo el ciclo no se completa, ya que esas larvas L1 procedentes de los huevos, al no hallarse en el hospedador propio, no podrán progresar hacia el estado adulto ni alcanzar la madurez sexual y, en consecuencia, serán eventualmente expulsadas del intestino, quedando el paciente totalmente libre del parásito.

Por el contrario, en su hospedador característico, los suidos, el ciclo es "directo", poniendo las hembras huevos sin embrionar que se expulsan con las heces, embrionando posteriormente en el medio ambiente externo si las condiciones son adecuadas, transmitiéndose a continuación a otros hospedadores a través de mecanismos de transmisión fecal-oral y eclosionando, tras la ingestión, en el último tramo del intestino delgado para liberar las larvas L1, que evolucionan hasta adultos sexualmente maduros tras haber superado las correspondientes mudas (L2, L3 y L4), con lo que se completa el ciclo.

Como se indica en el epígrafe siguiente, el hecho de que no se trate de un parásito humano tiene innumerables ventajas en cuanto a reducción de riesgos, tanto de ocurrencia de patología como de posibilidad de transmisión de enfermedades inter-humanos derivadas de la utilización de productos de T. suis. Sin embargo cabe preguntarse en este punto cómo el parásito puede producir efectos inmunorreguladores en el hombre si no ha co-evolucionado con él en busca de una solución adaptativa, que es uno de los condicionantes clave que justifican los efectos inmunorreguladores de algunos parásitos según la hipótesis de la higiene. En este sentido hay que apelar a la alta proximidad genética existente entre los suidos y los seres humanos, que ha hecho que las especies de la clase Adenophorea / orden Trichocephalida como T. suis y T. trichiura, que los colonizan respectivamente, resulten morfológicamente indistinguibles $^{(12)}$. Esta similitud hace que uno sea capaz de producir efectos inmunorreguladores propios de una adaptación coevolutiva sobre el sistema inmunológico del hospedador de la otra especie, pero con la ventaja de no producir un ciclo de infección completo.

Hay que decir que en el tratamiento de enfermedades autoinmunes también es viable el empleo de parásitos específicos de la especie humana como es Necator americanus, pero con las debidas precauciones. De hecho el caso más frecuente es éste, ya que para asegurar los efectos inmunorreguladores del parásito, éste ha debido de co-evolucionar 
con el ser humano, excepción hecha de T. suis, como se ha indicado antes.

En el caso de la utilización de Necator americanus en el tratamiento de la enfermedad de Crohn, para reducir los efectos secundarios al mínimo es necesario limitar la carga parasitaria ya que el aspecto patológico principal de este parásito es la anemia ferropénica debida condición hematófaga del mismo; y que se mantiene en el ámbito subclínico con cargas bajas de parásito. Es por ello que en la terapia helmíntica se está considerando la utilización de un máximo de 10 larvas de N. americanus ${ }^{(5)}$. En comparación con el tratamiento con T. suis hay que decir que los efectos terapéuticos con $N$. americanus presentan una mayor duración, requiriendo menor número de administraciones ${ }^{(5)}$. Este hecho puede estar relacionado, bien con que $N$. americanus está más intrínsecamente relacionado con el sistema inmunológico humano por haber co-evolucionado con él, mientras que T. suis no lo ha hecho, o bien debido a la mayor duración (1-2 años) de la infección por N. americanus en el intestino humano.

\section{Empleo farmacológico}

T. suis es poseedor de una serie de características que lo hacen un agente idóneo para su empleo terapéutico:

- No hay riesgo de que cause patología grave en el hombre, en el que no puede cerrar su ciclo biológico.

- Parasita principalmente a animales, suidos, lo que reduce enormemente su potencial intrínseco como transmisor de infecciones inter-humanos.

- Es altamente productivo: la hembra pone entre $5.000 \mathrm{y}$ 10.000 huevos / día, que es el estado que actualmente se está utilizando en terapéutica. En general se emplean dosis de 2.500 huevos cada dos o tres semanas con cada paciente en el tratamiento de enfermedades autoinmunes.

- Posee efectos inmunomoduladores.

En la figura 5, se detalla el proceso de obtención del biofármaco consistente en huevos de $T$. suis en el que como puede verse, los riesgos de transmisión de otras enfermedades o de falta de efectividad por inactivación del material biológico, son mínimos.

\section{Ensayos clínicos}

Tras la justificación teórica presentada en los epígrafes anteriores cabe preguntarse si todo ello se traduce en el tratamiento de enfermedades autoinmunes de un modo efectivo y seguro, punto que se trata en el presente epígrafe a través del análisis de ensayos clínicos.

Las limitaciones de los tratamientos actuales de las enfermedades autoinmunes han hecho que se busquencontinuamente nuevas terapias para reducir la sintomatología de estas enfermedades y prevenir exacerbaciones. Así por ejemplo actualmente se utilizan los siguientes tratamientos de las enfermedades inflamatorias intestinales, que no llegan a reducir suficientemente la sintomatología y morbilidad delas mismas:

- control riguroso de la dieta como medida preventiva.

- inmunosupresores (ciclosporina, azatioprina, 6-mercaptopurina, etc) y antiinflamatorios esteroideos, tanto vía sistémica como local, como medidas de control de las manifestaciones agudas,

- cirugía en los casos más severos y que no responden a los tratamientos anteriores.

$Y$ es precisamente en los casos refractarios de estas enfermedades al tratamiento habitual, en los que los tratamientos basados en helmintos han demostrado mayor eficacia, llegando incluso a la respuesta terapéutica en la mayoría (80\%) de pacientesde enfermedad de Crohnparticipantes en el correspondiente ensayo clínico ${ }^{(13)}$, ver datos resumidos en la tabla 2. En el 72,4\% de los casos incluso hubo remisión sintomatológica, lo que significa que los síntomas de la enfermedad desaparecieron durante el tratamiento. La efectividad en el caso del tratamiento de colitis ulcerativa, sin ser tan espectacular como en el caso de la enfermedad de Crohn, sí que muestra una respuesta al tratamiento significativa estadísticamente $(\mathrm{p}=0,04)^{(14)}$.

Figura 5. Proceso de elaboración, a partir de Trichurissuis, del biofármaco en investigación utilizado para tratar enfermedades autoinmunes. Elaboración propia a partir de la bibliografía consultada. PBS, Phosphate Buffered Saline (tampón fosfato salino)

\begin{tabular}{|c|c|c|c|c|c|c|c|}
\hline $\begin{array}{l}\text { Selección de } \\
\text { Animales }\end{array}$ & Infectación & Extracción & Cultivo in vitro & $\begin{array}{c}\text { Embrionamiento in } \\
\text { vitro }\end{array}$ & Axenización & Conservación & Preparación dosis \\
\hline Cerdos sanos & $\begin{array}{l}\text { Administración de } \\
\text { huevos de T.suis }\end{array}$ & $\begin{array}{l}\text { De los nematodos } \\
\text { quirúrgicamente }\end{array}$ & $\begin{array}{l}\text { Se cultivan in vitro } \\
\text { sin problema }\end{array}$ & $\begin{array}{l}5 \text { a } 6 \text { semanas en } \\
\text { PBS a } 22^{\circ} \mathrm{C} \text { con: } \\
\text { - Estreptomicina } \\
\text { - } \text { Anfotericina B } \\
\text { - Penicilina }\end{array}$ & $\begin{array}{l}\text { - Una vez } \\
\text { embrionados, en } \\
\mathrm{K}_{2} \mathrm{Cr}_{2} \mathrm{O}_{7} 0,2 \% \\
\text { - Lavado en } \\
\text { solución salina. }\end{array}$ & $\begin{array}{l}\text { - En PBS a } 5^{\circ} \mathrm{C} \\
\text { - Se realizan } \\
\text { cultivos estándar } \\
\text { periódicamente } \\
\text { - Se verifica su } \\
\text { viabilidad a través } \\
\text { de la infección de } \\
\text { cerdos } \\
\text { - Duración unos } 9 \\
\text { meses }\end{array}$ & $\begin{array}{l}\text { Mediante conteo } \\
\text { en cámara } \\
\text { McMaster para } \\
\text { huevos de } \\
\text { helmintos (dosis } \\
\text { de } 2.500 \text { ) }\end{array}$ \\
\hline
\end{tabular}


En esta revisión se han analizado varios ensayos clínicos sobre enfermedades inflamatorias intestinales como son enfermedad de Crohn y colitis ulcerativa, en los que la terapia se ha basado en la administración de huevos de Trichuris suis a los pacientes. A continuación se resumen dos en-

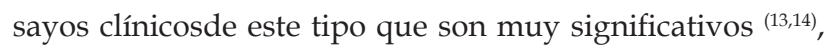
realizados entre 2001 y 2004, que se han escogido debido a la fiabilidad de los resultados publicados y a la madurez de los mismos. También se ha analizado un ensayo clínico de 2013en el que se emplea Trichuris suis para el tratamiento de esclerosis múltiple, aún sin resultados definitivos publicados ${ }^{(15)}$, para ilustrar cómo hoy en día se siguen realizando ensayos clínicos utilizando el mismo tipo de tratamiento que en los primeros citados, debido a sus buenos resultados y a su potencial.

Tabla 2. Resumen de resultados deensayos clínicos ${ }^{(13,14,15)}$.

\begin{tabular}{|c|c|c|c|c|c|}
\hline CENTRO & ENFERMEDAD & DISEÑO ENSAYO & TERAPIA & EFICACIA & SEGURIDAD \\
\hline $\begin{array}{l}\text { Universidad } \\
\text { Iowa, } 2003\end{array}$ & E. Crohn & $\begin{array}{l}29 \text { pacientes, abierto, } \\
\text { controlado contra place- } \\
\text { bo. Evaluación a través } \\
\text { del índice CDAI }\end{array}$ & $\begin{array}{l}2.500 \text { huevos de } \\
\text { T. suis cada } 3 \text { se- } \\
\text { manas durante } \\
24 \text { semanas. Sin } \\
\text { interrupción de } \\
\text { la medicación } \\
\text { habitual. }\end{array}$ & $\begin{array}{l}79,3 \% \text { de respuesta terapéu- } \\
\text { tica, } 72,4 \% \text { de remisión de } \\
\text { síntomas. Mejor resultado en } \\
\text { enfermedades de curso largo } \\
\text { y refractario. Sinergias de } \\
\text { asociación con la medicación } \\
\text { habitual. }\end{array}$ & $\begin{array}{l}\text { Sin aconte- } \\
\text { cimientos } \\
\text { adversos }\end{array}$ \\
\hline $\begin{array}{l}\text { Universidad } \\
\text { Iowa, } 2004\end{array}$ & Colitis Ulcerativa & $\begin{array}{l}54 \text { pacientes, doble } \\
\text { ciego, controlado contra } \\
\text { placebo. Evaluación a } \\
\text { través del índice IAE }\end{array}$ & $\begin{array}{l}2.500 \text { huevos de } \\
\text { T. suis cada } 2 \text { se- } \\
\text { manas durante } \\
12 \text { semanas. }\end{array}$ & $\begin{array}{l}44 \% \text { de respuesta terapéu- } \\
\text { tica, sin remisiones signifi- } \\
\text { cativas. Mejor resultado en } \\
\text { enfermedades de curso largo } \\
\text { y refractario. }\end{array}$ & $\begin{array}{l}\text { Sin aconte- } \\
\text { cimientos } \\
\text { adversos }\end{array}$ \\
\hline $\begin{array}{l}\text { Charité - Uni- } \\
\text { versitätsmedi- } \\
\text { zinBerlin, } 2013\end{array}$ & $\begin{array}{l}\text { Esclerosis múl- } \\
\text { tiple }\end{array}$ & $\begin{array}{l}50 \text { pacientes, doble } \\
\text { ciego, controlado contra } \\
\text { placebo 1:1. Evaluación } \\
\text { a través de análisis de } \\
\text { nuevas lesiones con } \\
\text { MRI e indicadores } \\
\text { clínicos. }\end{array}$ & $\begin{array}{l}2.500 \text { huevos de } \\
\text { T. suis cada } 2 \text { se- } \\
\text { manas durante } \\
12 \text { meses. }\end{array}$ & \multicolumn{2}{|c|}{$\begin{array}{l}\text { Resultados pendientes de publicar en el } \\
\text { momento de escribir el artículo. }\end{array}$} \\
\hline
\end{tabular}

CDAI (Crohn DiseaseActivityIndex), IAE (Índice de Actividad de la Enfermedad), MRI (MagneticResonanceImaging).

La menor efectividad en el tratamiento de la colitis ulcerativa frente a la enfermedad de Crohn puede apuntar hacia una menor proporción del componente Th1 y Th17 en la colitis ulcerativa frente a la enfermedad de Crohn, en beneficio de otros componentes de la respuesta inmunológica responsable de la enfermedad, por ejemplo el Th2. Por ello en colitis ulcerativa, a pesar de que la respuesta al tratamiento con T. suis es efectivo y significativo, podría encontrarse un parásito que regule el espectro de respuesta Th1/ Th17/Th2 de la enfermedad de un modo más eficiente para reducir el daño a tejidos y la inflamación en este caso. Esto es perfectamente viable ya que los efectos inmunorreguladores de los diferentes parásitos se basan en diferentes mecanismos que regulan de un modo diferente las respuestas de la inmunidad adaptativa.

A pesar de la importanciade la búsqueda de un parásito concreto que pueda equilibrar el espectro específico de respuesta inmunológica Th1/Th17/Th2/Treg/... causante de cada enfermedad, hay que decir que, cada parásito inmunorregulador presenta unos efectos sistémicos en todo el organismo y no solo en los órganos afectados por la enfermedad, que los hace útiles en el tratamiento de diferentes enfermedades de origen autoinmune sin aparente relación entre ellas. Así Trichuris suis se ha empleado en el tratamiento de enfermedades aparentemente tan alejadas de las inflamatorias intestinales como es la esclerosis múltiple ${ }^{(15)}$. Esta generalidad autoinmune es útil en el tratamiento de enfermedades de este tipo, por venir acompañadas generalmente de otras sintomatologías concomitantes de origen indudablemente autoinmune y que afectan a otros órganos diferentes del órgano diana de la enfermedad. Es más, estos hallazgos en cuanto a similar respuesta terapéutica a este tipo de tratamientos en esclerosis múltiple y enfermedades inflamatorias intestinales, ayudarán a desvelar aspectos del sistema inmunológico desconocidos hasta ahora y a ensayar tratamientos convencionales de algunas de estas enfermedades en las otras ${ }^{(16)}$, debido a la fuerte correlación fisiopatológica desvelada a través de una respuesta similar frente al tratamiento helmíntico.

Es por ello que las terapias basadas en helmintos suponen una gran promesa no sólo en el tratamiento de enfermedades, sino también en su investigación. Ya que una buena respuesta al tratamiento helmíntico de una enfermedad como es el autismo, puede confirmar la relevancia del com- 
ponente inmunológico como uno de los factores causantes de la misma.

\section{DISCUSIÓN}

A pesar de lo prometedor de las terapias helmínticas hay que decir que no todos los parásitos son inmunorreguladores y, por lo tanto aptos para el tratamiento de enfermedades autoinmunes. Por el contrario muchos producen enfermedades inflamatorias y alérgicas. Es más, dentro de los inmunorreguladores no todos son útiles en el tratamiento de cualquier enfermedad autoinmune, ni siquiera para cualquier individuo afectado por una misma enfermedad de este tipo, existiendo una variabilidad importante respecto al individuo y a los condicionantes de su enfermedad concreta. Es posible que toda esta variabilidad dependa del perfil concreto de respuesta inmunológica Th1/Th17/Th2/ Treg/ ...de cada caso, sin embargo no se conoce con certeza en qué medida; la hipótesis de la higiene no ha pasado de ser una hipótesis y es necesaria más investigación al respecto. Es por ello que actualmente no existen terapias helmínticas aprobadas por las principales administraciones del medicamento para su uso fuera de la investigación, si bien lo prometedor de estas terapias ha traído consigo la autorización de algunas de las mismas como "productos en fase de investigación clínica" por parte de estas administraciones, como son la European Medicines Agency (EMA) o la Federal Drug Administration (FDA) de los Estados Unidos.

Esta investigación adicional necesaria es difícilmente justificable desde el punto de vista económico cuando se trata de biofármacos consistentes en fases de parásitos como son los huevos, con las dificultades de patente que ello presenta ${ }^{(17)}$. Por eso será clave el desarrollo de fármacos sintéticos derivados de las biomoléculas ${ }^{(18)}$ de los parásitos responsables de las acciones terapéuticas estudiadas, de más fácil patente que los productos biológicos como los huevos de T. suis y, además, soslayando algunos problemas éticos que se plantean en relación con estas terapias.Esta mayor facilidad de patente viabilizará el caso de negocio para la realización de las costosas inversiones en ensayos clínicos que son necesarias en este campo. Algunos de estos fármacos sintéticos ya se están ensayando actualmente ${ }^{(11)}$, como se ha explicado anteriormente. Sin embargo hoy en díaaún se está lejos de la identificación del conjunto de biomoléculas de cada parásito que son suficientes para ejercer las acciones buscadas.

Por otro lado hay que indicar que el diseño de estos fármacos derivados de biomoléculas, al eliminar los riesgos de infección parasitaria y de contagio de otras infecciones de las que el material parasitario puede ser vector, posibilitará la superación de las barreras de orden ético sobre si es lícitocorrer el riesgo de infectar a un paciente para tratarle de una enfermedad. Se ha demostrado tanto en los ensayos clínicos realizados ${ }^{(13,14,15)}$ como en otras experiencias citadas en (19), que el tratamiento con Trichuris suis es altamente seguro por no cerrarse el ciclo biológico en el hombre. Por otro lado el proceso tecnológico de preparación del material terapéutico, figura 5, hace que el riesgo de que éste pueda ser agente transmisor de otras enfermedades sea despreciable. Si a esto se añade que $T$. suis no es un parásito del ser humano, las posibilidades de vehicular enfermedades interhumanos son prácticamente nulas.

Además de los indicados anteriormente, otros próximos pasos consistirán en seguir abriendo el abanico de enfermedades a tratar a través de la terapia helmíntica, vista la involucración de disfunciones del sistema inmunológico en muchas enfermedades aparentemente no relacionadas con el mismo, como es el ejemplo del autismo ${ }^{(7)}$ o la aterosclerosis $^{(5)}$.

\section{REFERENCIAS}

1. Leslie M, Horner A. Gut microbes keep rare immune cells in line. Science. 2012; 335:1428.

2. The Naked Scientists ${ }^{\circledR}$ [internet]. Cambridge University; c2000-2014. "The Hygiene Hypothesis Unraveled. Gut-dwelling parasites may protect us against potentially deadly diseases"; 2013 [cited 24th Feb 2014]; Available from http:// www.thenakedscientists.com/HTML/articles/article/thehygiene-hypothesis-unraveled/.

3. Elliott DE and Weinstock JV. Helminth-host immunological interactions: prevention and control of immune-mediated diseases. Ann NY Acad Sci. 2012; 1247:83-96.

4. Khan AR, Fallon PG. Helminth therapies: Translating the unknown unknowns to known knowns. Int J Parasitol. 2013; 43(3-4):293-299.

5. Reddy A, Bernard Fried B. An update on the use of helminths to treat Crohn's and other autoimmunune diseases. Parasitol Res. 2009; 104:217-221.

6. Siniscalco D, Antonucci N. Possible use of Trichuris suis ova in autism spectrum disorders therapy. Med Hypotheses. 2013; 81:1-4.

7. Heberling CA, Dhurjati PS, Sasser M. Hypothesis for a systems connectivity model of autism spectrum disorder pathogenesis: Links to gut bacteria, oxidative stress, and intestinal permeability. Med Hypotheses. 2013; 80:264-270.

8. Girgis N, Gundra U, Loke P. Immune regulation during helminth Infections. Plos Pathog. 2013; 9(4):1-3.

9. Rzepecka J et Al. Small molecule analogues of the immunomodulatory parasitic helminth product ES-62 have anti-allergy properties. Int J Parasitol. 2014; 44:669-674.

10. Klaver EJ, Kuijk LM, Laan LC, Kringel H, van Vliet SJ, Bouma G, Cummings RD, Kraal G, van Die I. Trichuris suis-induced 
modulation of human dendritic cell function is glycan-mediated. Int J Parasitol. 2013; 43(3-4):191-200.

11. Finlay CM, Walsh KP, Mills KH. Induction of regulatory cells by helminth parasites: Exploitation for the treatment of inflammatory diseases. Immunol Rev. 2014; 259(1):206-230.

12. Cutillas C, Callejón R, de Rojas M, Tewes B, Ubeda JM, Ariza C, Guevara DC, Trichuris suis and Trichuris trichiura are different nematode species. Acta Trop. 2009; 111:299-307.

13. Summers RW, Elliott DE, Urban JF, Thompson R, Weinstock JV. Trichuris suis therapy in Crohn's disease. Gut. 2005; 54:8790.

14. Summers RW, Elliott DE. Trichuris suis therapy for active ulcerative colitis: A randomized controlled trial. Gastroenterology. 2005; 128:825-832.

15. Rosche B, Wernecke K, Ohlraun S, Dörr J and Paul F. Trichuris suis ova in relapsing-remitting multiple sclerosis and clinically isolated syndrome (TRIOMS): study protocol for a randomized controlled trial. Trials. 2013; 14:112.

16. Hsing Lin C, Kadakia S, Frieri M. New insights into an autoimmune mechanism, pharmacological treatment and relationship between multiple sclerosis and inflammatory bowel disease. Autoimmun Rev. 2014; 13:114-116.

17. Prerequisites for the pharmaceutical industry to develop and commercialise helminths and helminth-derived product therapy. Int J Parasitol. 2013; 43:323.

18. Reddy MVR. Immunomodulators of helminthes: promising therapeutics for autoimmune disorders and allergic diseases. Indian J Clin Biochem. 2010; 25(2):110.
19. Organización Panamericana de la salud. Zoonosis y enfermedades comunes al hombre y a los animales. $3^{\mathrm{a}}$ edición, Washington: Organización Panamericana de la Salud; 2003.

20. Panackel C, Sebastian B, Mathai S, Thomas R. Trichuris dysentery syndrome. Ann Trop Med Public Health. 2011; 4(2):148149.

21. Summers R, Elliott D, Weinstock J. Therapeutic colonization with Trichuris suis. Arch Pathol Lab Med. 2006; 130(12):17531754.

22. Hepworth MR, Hamelmann E, Lucius R, Hartmann S. Looking into the future of Trichuris suis therapy. J Allergy and Clinical Immunology. 2010; 125(3):767-768.

23. Elliott DE and Weinstock JV. Where are we on worms?. Curr Opin Gastroen. 2012; 28(6):551-556.

24. Wolff MJ, Broadhurst MJ, Loke P. Helminthic therapy: improving mucosal barrier function. Trends Parasitol. 2012; 28(5):187.

25. Rook GA. Hygiene Hypothesis and autoimmune diseases. Clin Rev Allerg Immu. 2012; 42:5 - 15.

26. Chelsea ME, McDougall JJ, Sharkey KA and McKay DM. Helminth parasites and the modulation of joint inflammation. Journal Parasitol Res. 2011.

27. Grant B. Opening a Can of Worms. The Scientist. 2011; 25(2):42-47.

28. Becker KG. Autism, asthma, inflammation, and the hygiene hypothesis. Med Hypotheses. 2007; 69:731-740. 